$\triangle$ Andrii Shyshatskyi, PhD, Senior Researcher, Research Department of Electronic Warfare Development, Central Scientific Research Institute of Armament and Military Equipment of the Armed Forces of Ukraine, Kyiv, Ukraine, e-mail: ierikon13@gmail.com, ORCID: https://orcid.org/0000-0001-6731-6390

Volodymyr Ovchynnyk, Lecturer, Department of Armored Vehicles, Odessa Military Academy, Odesa, Ukraine, ORCID: https://orcid.org/ 0000-0001-7653-7136

Andrii Momotov, Department of Construction and Road-Building Machinery, Kharkiv National Automobile and Highway Uni- versity, Kharkiv, Ukraine, ORCID: https://orcid.org/0000-00015997-4561

Nadiia Protas, PhD, Associate Professor, Department of Information Systems and Technologies, Poltava State Agrarian University, Poltava, Ukraine, ORCID: https://orcid.org/0000-0003-0943-0587

Andriy Solomakha, Senior Lecturer, Department of Military Training, The Bohdan Khmelnytsky National University of Cherkasy, Cherkasy, Ukraine, ORCID: https://orcid.org/0000-0001-7390-4156

$\triangle$ Corresponding author

UDC 629.7.615.3

DOI: $10.15587 / 2706-5448.2022 .252712$

Article type «Reports on Research Projects»

Natalja Ashhepkova

\title{
ANALYSIS OF THE INERTIA TENSOR OF AUTONOMOUS MOBILE ROBOT
}

The object of research is the inertia tensor of an autonomous mobile robot (AMR) with a manipulator with different configurations of their mutual position. As an example of the AMR design of a changing configuration, an all-wheel drive four-wheeled platform with a manipulator is considered, consisting of a docking disk rotating around a vertical axis and rod links of the arm connected by rotational kinematic pairs of the fifth class. The mass of moving structural elements, i. e., a manipulator with a load, is 10-20\% of the mass of the robot platform. Let's consider that the links of the manipulator and the platform are absolutely rigid and homogeneous bodies with a constant density; let's neglect the mass of kinematic pairs. The next step in the analysis of the AMR inertia tensor of a changing configuration can be a study taking into account the elastic properties of the manipulator links, the uneven distribution of the masses of the platform, and the characteristics of the kinematic pairs.

The dependence of the values of the elements of the AMR inertia tensor of a changing configuration on the values of the generalized coordinates of the moving elements of the structure and the ratio of the mass of the platform and the mass of the moving elements of the structure has been studied. The analysis of the inertia tensor of the AMR with a manipulator at different configurations of their mutual position showed that the values of the centrifugal moments of inertia of the system during the relative motion of the manipulator are commensurate with the value of the axial moments of inertia of the system, even if the mass of the moving structural elements is less than $10 \%$ of the mass of the platform. In most existing AMRs, the mass of moving structural elements is up to $20 \%$ of the platform mass, therefore, in the general case, the inertia tensor of such a system should be taken as off-diagonal and non-stationary. In the future, this will make it possible to refine the equation of dynamics, take into account the relationship of control channels, simulate the movement of AMR of a changing configuration, and optimize energy costs.

Since AMR with the manipulator is an example of the «changing $A M R \gg$ object class, the results obtained can be applied to all objects of this class.

Keywords: autonomous mobile robot, manipulator, moment of inertia, off-diagonal and non-stationary inertia tensor.

\section{How to cite}

Ashhepkova, N. (2022). Analysis of the inertia tensor of autonomous mobile robot. Technology Audit and Production Reserves, 1 (2 (63)), $36-40$. doi: http:// doi.org/10.15587/2706-5448.2022.252712

\section{Introduction}

In robotics, there is a tendency to increase the autonomy of mobile robotic systems (RS). The works [1,2] emphasize the importance of using the modular principle of assembling the structures of autonomous mobile robots (AMRs). The introduction of the modular principle leads to the creation of flexible functional reconfigured complexes with the possibility of further modernization. Replacing modules in AMR designs allows to timely send the RS to solve a new problem when the operational environment or working space conditions change, quickly carry out current repairs and extend the service life. The expansion of the scope and complication of tasks for modern AMR determines 
the presence of structural elements moving in relation to the platform [3]. An example of such structural elements can be a manipulator, executive bodies of specialized equipment or mobile attachments: probe, storms, bucket, etc. During the operation of the AMR, these elements move relative to the platform, changing the geometry of the masses of the system.

In remote work in extreme a priori uncertain conditions, when performing technological operations with fragile or explosive objects, AMR with a manipulator is used [1-3]. To study the dynamics, such AMR of a changing configuration can be considered as a manipulator on a movable base or a system of a carrier and a transferred body. The presence of a movable base increases the maneuverability of the handling AMR, expands the working space and increases the number of permissible configurations of the manipulator, but reduces the positioning accuracy of the gripper (attached equipment or tool). Accounting for inertial characteristics when compiling the equations of dynamics makes it possible to provide an optimal structure [4] or to investigate the contact forces of the manipulator [5]. The results of the study for a spacecraft with a manipulator are given in $[6,7]$. It was proved in [6] that taking into account the off-diagonality and non-stationarity of the control object makes it possible to reduce the energy consumption of the carrier system and the transferred body by $13-50 \%$ for various angular maneuvers. In [7], the control of a space manipulator was studied to achieve a given performance and speed.

To overcome the consequences of man-made and natural disasters for work in extreme a priori uncertain conditions, it is advisable to apply coordinated actions of the AMR group [8-10]. At the same time, the control system of each AMR ensures movement to a given point in the working space and bypassing obstacles [8]. The most common drawback of existing AMR control methods is the inconsistency of control algorithms with kinematic constraints and the dynamics of individual robots [9]. In [10], it is noted that complex large-scale operations are coupled with a long stay of the AMR in the working area with a limited battery capacity. To ensure the successful functioning of the group, it is necessary to implement the «watch» mode of operation of the AMR, in other words, the work alternately leaves the working area for recharging. In this case, one should take into account the parametric and functional heterogeneity of the AMR within the group, which ensures the maximum capabilities of the detachment.

When moving an AMR of a changing configuration along a given route, the control system must ensure the minimum deviation of the center of mass of the platform from the given trajectory [9]. In this case, the control moments are directed along the axes of the coordinate system coupled with the AMR platform. In the case of relative motion of the AMR structural elements of a changing configuration, its main central axes of inertia do not coincide with the axes of the coordinate system coupled with the AMR platform. As a consequence, the AMR inertia tensor in the coordinate system coupled with the platform is off-diagonal and non-stationary. In many cases, this is neglected in the analysis of the system dynamics and the formation of control moments, taking the off-diagonal elements small without estimating their values.

The relevance of the work lies in the need to analyze the inertia tensor of the AMR with a manipulator with different configurations of their mutual position. Based on the data obtained, it is possible to establish the dependence of the values of the elements of the AMR tensor of a changing configuration on the values of the generalized coordinates of the moving structural elements, the ratio of the mass of the AMR platform and the mass of the movable structural elements.

Thus, the object of research is the inertia tensor of the AMR with a manipulator with different configurations of their mutual position. The aim of research is to analyze the off-diagonality and non-stationarity of the AMR inertia tensor of a changing configuration.

\section{Research methodology}

In order to create flexible multifunctional reconfigured complexes with the possibility of further modernization, let's believe that the AMR design with a changing configuration is based on a modular principle. As an example of the AMR design of a changing configuration, let's consider an all-wheel drive 4-wheeled platform with a manipulator, consisting of a docking disk rotating around a vertical axis, and rod links of the arm connected by rotational kinematic pairs of the fifth class (Fig. 1).

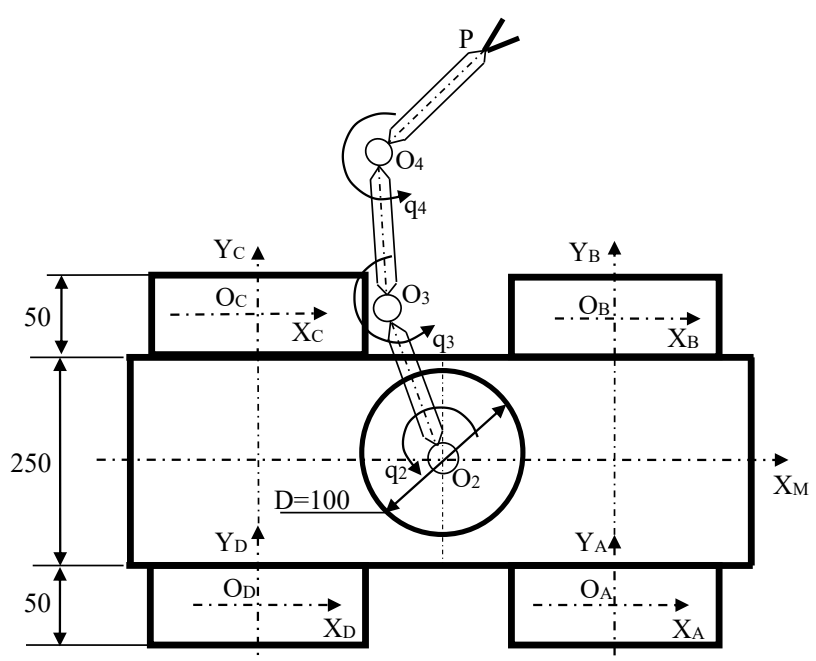

Fig. 1. Calculation model of AMR with a changing configuration

Let's introduce the following right-handed Cartesian coordinate systems (Fig. 2):

1) $O_{0} X_{0} Y_{0} Z_{0}$ - inertial coordinate system;

2) $M X_{M} Y_{M} Z_{M}$ - moving base coordinate system, the origin of which is connected with the center of mass of the platform by point $M$. The axes coincide with the main central axes of inertia of the platform:

- $M Z_{M}$ axis is perpendicular to the platform movement plane, coincides with the local vertical and is directed upwards to the manipulator docking disk;

- axis $M X_{M}$ is located in the plane of movement of the platform and is directed in the direction of movement; - $M X_{M}$ axis is located in the plane of movement of the platform and complements the system to the right one;

3) $O_{1} X_{1} Y_{1} Z_{1}$ - coupled moving coordinate system, the origin of which is the center of mass of the docking disk, point $O_{1}$, and the axes coincide with the main central axes of inertia of the disk. In the initial position of the docking disk, the corresponding axes of the $O_{1} X_{1} Y_{1} Z_{1}$ and $M X_{M} Y_{M} Z_{M}$ coordinate systems are parallel; 
4) $O_{i} X_{i} Y_{i} Z_{i}(i=2,3,4)$ - coupled moving coordinate systems, the origin of which are the centers of kinematic pairs of the point $O_{i}$. The $O_{i} X_{i}$ axes coincide with the longitudinal axes of the rod links of the manipulator arm and are directed towards the point $O_{i-1}$. In the initial position of the manipulator links, the corresponding axes of the $O_{i} X_{i} Y_{i} Z_{i}$ and $M X_{M} Y_{M} Z_{M}$ coordinate systems are parallel.

To determine the calculations, let's assume that the mass of the manipulator with the load is up to $20 \%$ of the mass of the AMR platform. The introduction of the modular design principle of the AMR determines the design features of the manipulator. The first link of the manipulator (docking disk) is a disk with a diameter of $100 \mathrm{~mm}$ and a height of $50 \mathrm{~mm}$; the second, third and fourth links are rods with a diameter of $30 \mathrm{~mm}$, length $\mathrm{O}_{4} \mathrm{P}=150 \mathrm{~mm}, \mathrm{O}_{3} \mathrm{O}_{4}=150 \mathrm{~mm}, \mathrm{O}_{2} \mathrm{O}_{3}=250 \mathrm{~mm}$. The AMR platform has the shape of a parallelepiped (Fig. 3).

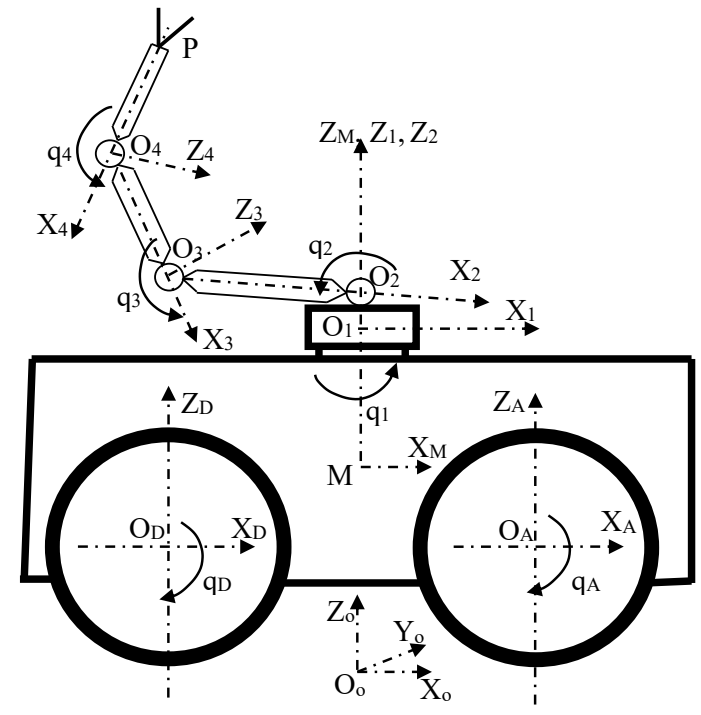

Fig. 2. Scheme of coordinate systems
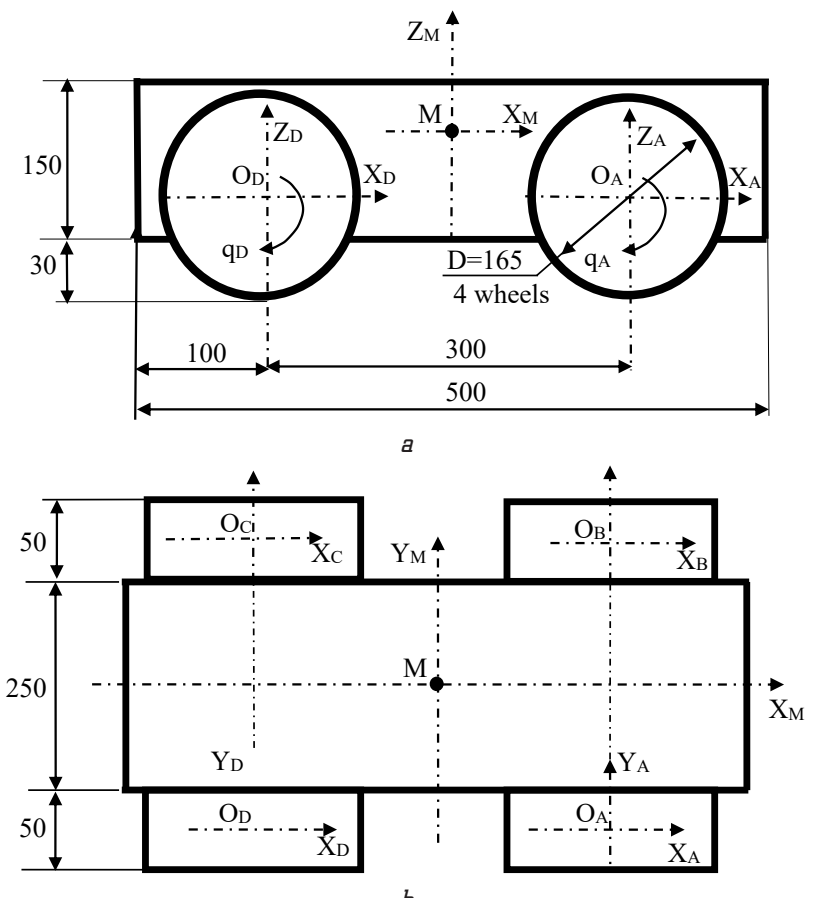

Fig. 3. Calculation model of the autonomous mobile robot platform: $a$ - front view; $b$ - top view
The generalized coordinates have design limitations:

$$
\begin{aligned}
& q_{1} \in\left[0 ; 350^{\circ}\right], \quad q_{2} \in\left[10 ; 170^{\circ}\right], \\
& q_{3} \in\left[0 ; 120^{\circ}\right], \quad q_{4} \in\left[0 ; 120^{\circ}\right] .
\end{aligned}
$$

Let's consider that the manipulator links and the platform are absolutely solid and homogeneous bodies with a density of $2.7 \mathrm{~g} / \mathrm{cm}^{3}$. Let's neglect the mass of kinematic pairs. The motor-wheel has a diameter of $165 \mathrm{~mm}$, a thickness of $50 \mathrm{~mm}$ and a mass of $2800 \mathrm{~g}$.

AMR with the manipulator is a complex mechanical system that can be considered a manipulator on a moving base. The links of the manipulator are the transferred bodies, the platform is the carrier [11]. The moments of inertia of each link are functions of the generalized coordinates [12], but the dependence of the elements of the inertia tensor of the AMR with a manipulator on the values of the generalized coordinates has not been established.

With the assumption that all links are absolutely rigid bodies, AMR with a manipulator is a dynamic system of undeformed bodies with nonholonomic stationary constraints.

To detect the inertia tensor of the system, the moments of inertia of its components (platform, wheels, manipulator and load) were determined in coupled moving coordinate systems. The movement of each transferred body relative to the carrier was presented as a sequence of rotations and parallel transfers of the corresponding moving coordinate system $O_{i} X_{i} Y_{i} Z_{i}(i=1,2,3,4)$ with respect to the base coordinate system $M X_{M} Y_{M} Z_{M}$ [11]. By means of the angle matrix for each rotational kinematic pair, coordinate transformation formulas and the Steiner theorem, the elements of the inertia tensor of each transferred body in the base coordinate system were calculated. The moments of inertia of the AMR of a changing configuration relative to the axes of the coupled coordinate system were calculated by summing the moments of inertia of the parts of the system [11, 12].

\section{Research results and discussion}

Let's assume that the laws of change of the generalized coordinates of the manipulator links $q_{1}(t), q_{2}(t), q_{3}(t), q_{4}(t)$ are known; the initial positions of the manipulator links $q_{1}(t), q_{2}(t), q_{3}(t), q_{4}(t)$; and the coordinates of the gripper pole at the initial time $P(15 ; 0 ; 0)$ in the coordinate system $O_{4} X_{4} Y_{4} Z_{4}$. It is necessary to determine the dependence of the value of the elements of the AMR tensor of the changing configuration on the values of the generalized coordinates of the moving elements of the structure. It is advisable to evaluate the dependence of the tensor elements on the ratio of the mass of the AMR platform and the mass of the moving elements of the structure. Based on the data obtained, let's analyze the off-diagonality and non-stationarity of the AMR inertia tensor of a changing configuration.

The moments of inertia of the AMR platform with respect to the base coordinate system with the indicated dimensions (Fig. 1) and the mass of the platform $50.625 \mathrm{~kg}$ are: $I x x=3.586106 \mathrm{~g} \cdot \mathrm{cm}^{2} ; \quad I y y=1.15107 \mathrm{~g} \cdot \mathrm{cm}^{2}$; $I z z=1.318107 \mathrm{~g} \cdot \mathrm{cm}^{2} ; I x y=I x z=I y z=0$.

Let's calculate the moments of inertia of each link of the AMR manipulator with respect to the coordinate system coupled with the link using the Mathcad application package. The calculation results are presented in Table 1. 
Table 1

Moments of inertia of manipulator links in coupled coordinate systems

\begin{tabular}{|c|c|c|c|c|}
\hline \multirow{2}{*}{$\begin{array}{c}\text { Coupled coor- } \\
\text { dinate system }\end{array}$} & First link & Second link & Third link & Fourth link \\
\cline { 2 - 5 } & $O_{1} X_{1} Y_{1} Z_{1}$ & $O_{2} X_{2} Y_{2} Z_{2}$ & $O_{3} X_{3} Y_{3} Z_{3}$ & $O_{4} X_{4} Y_{4} Z_{4}$ \\
\hline$I x x, \mathrm{~g} \cdot \mathrm{cm}^{2}$ & $1.325 \cdot 10^{4}$ & 106.029 & 63.617 & 63.617 \\
\hline$I y y, \mathrm{~g} \cdot \mathrm{cm}^{2}$ & $1.546 \cdot 10^{4}$ & $4.423 \cdot 10^{4}$ & $9.574 \cdot 10^{3}$ & $9.574 \cdot 10^{3}$ \\
\hline$I z z, \mathrm{~g} \cdot \mathrm{cm}^{2}$ & $1.546 \cdot 10^{4}$ & $4.423 \cdot 10^{4}$ & $9.574 \cdot 10^{3}$ & $9.574 \cdot 10^{3}$ \\
\hline$I x y, \mathrm{~g} \cdot \mathrm{cm}^{2}$ & 0 & 0 & 0 & 0 \\
\hline$I x y, \mathrm{~g} \cdot \mathrm{cm}^{2}$ & 0 & 0 & 0 & 0 \\
\hline$I x y, \mathrm{~g} \cdot \mathrm{cm}^{2}$ & 0 & 0 & 0 & 0 \\
\hline
\end{tabular}

The movement of each link of the manipulator with respect to the AMR platform can be represented as a sequence of turns and parallel transfers [3-5].

For each rotational kinematic pair, let's obtain the matrices of angles:

$$
\begin{aligned}
& Q_{3}^{4}=\left[\begin{array}{ccc}
q_{4} & 90^{\circ} & 90^{\circ}-q_{4} \\
90^{\circ} & 0^{\circ} & 90^{\circ} \\
90^{\circ}+q_{4} & 90^{\circ} & q_{4}
\end{array}\right] ; \\
& Q_{2}^{3}=\left[\begin{array}{ccc}
q_{3} & 90^{\circ}-q_{3} & 90^{\circ} \\
90^{\circ}+q_{3} & q_{3} & 90^{\circ} \\
90^{\circ} & 90^{\circ} & 0^{\circ}
\end{array}\right] ; \\
& Q_{1}^{2}=\left[\begin{array}{ccc}
q_{2} & 90^{\circ} & 90^{\circ}-q_{2} \\
90^{\circ} & 0^{\circ} & 90^{\circ} \\
90^{\circ}+q_{2} & 90^{\circ} & q_{2}
\end{array}\right] ; \\
& Q_{M}^{1}=\left[\begin{array}{ccc}
q_{1} & 90^{\circ}-q_{1} & 90^{\circ} \\
90^{\circ}+q_{1} & q_{1} & 90^{\circ} \\
90^{\circ} & 90^{\circ} & 0^{\circ}
\end{array}\right] .
\end{aligned}
$$

Taking into account the angular transformation of coordinates and using the Steiner theorem [1], let's calculate the elements of the AMR inertia tensor of a changing configuration with respect to the coupled coordinate system $M X_{M} Y_{M} Z_{M}$ using the Mathcad application package. The results of calculations of the values of the elements of the inertia tensor depending on the values of the generalized coordinates of the moving elements of the structure are given in Table 2. Last column of Table 2 shows the values of the elements of inertia for the load, the mass of which is less than $10 \%$ of the mass of the AMR platform.

Using the Mathcad application package, let's calculate the dependence of the elements of the AMR inertia tensor of a changing configuration on the ratio of the mass of the AMR platform and the mass of the moving elements of the structure. Results of calculations in the coupled coordinate system $M X_{M} Y_{M} Z_{M}$ are given in Table 3 .

As a result of the study, it is possible to see (Tables 2, 3) that the AMR inertia tensor of a changing configuration in the base coordinate system is off-diagonal and nonstationary. A feature of objects of this class is the commensurability of centrifugal and axial moments of inertia with respect to the axes coupled with the AMR platform of the coordinate system. The values of the elements of the AMR tensor of the changing configuration depend non-linearly on the values of the generalized coordinates of the moving elements of the structure (Table 2).
Table 2

\begin{tabular}{|c|c|c|c|}
\hline $\begin{array}{c}\text { The value of } \\
\text { the generalized } \\
\text { coordinates of the } \\
\text { manipulator }\end{array}$ & $\begin{array}{l}\text { AMR platform } \\
\text { with wheels }\end{array}$ & $\begin{array}{l}\text { AMR platform } \\
\text { with wheels and } \\
\text { manipulator }\end{array}$ & $\begin{array}{l}\text { AMR platform } \\
\text { with wheels, } \\
\text { manipulator and } \\
\text { loading }\end{array}$ \\
\hline $\begin{array}{c}q_{1}=0 ; q_{2}=10 ; \\
q_{3}=0 ; q_{4}=0\end{array}$ & $\begin{array}{c}I x x=6.544 \cdot 10^{6} \\
I y y=1.407 \cdot 10^{7} \\
I z z=1.822 \cdot 10^{7} \\
I x y=0 \\
I y z=0 \\
I x z=0\end{array}$ & $\begin{array}{l}I x x=1.532 \cdot 10^{7} \\
I y y=5.123 \cdot 10^{7} \\
I z z=4.661 \cdot 10^{7} \\
I x y=1.384 \cdot 10^{7} \\
I y z=2.906 \cdot 10^{7} \\
I x z=6.421 \cdot 10^{6}\end{array}$ & $\begin{array}{l}I x x=3.518 \cdot 10^{7} \\
I y y=8.282 \cdot 10^{7} \\
I z z=5.835 \cdot 10^{7} \\
I x y=1.384 \cdot 10^{7} \\
I y z=2.906 \cdot 10^{7} \\
I x z=2.168 \cdot 10^{7}\end{array}$ \\
\hline $\begin{array}{c}q_{1}=90 ; q_{2}=10 ; \\
q_{3}=0 ; q_{4}=0\end{array}$ & $\begin{array}{c}I x x=6.544 \cdot 10^{6} \\
I y y=1.407 \cdot 10^{7} \\
I z z=1.822 \cdot 10^{7} \\
I x y=0 \\
I y z=0 \\
I x z=0\end{array}$ & $\begin{array}{l}I x x=1.531 \cdot 10^{7} \\
I y y=5.115 \cdot 10^{7} \\
I z z=4.661 \cdot 10^{7} \\
I x y=1.392 \cdot 10^{7} \\
I y z=2.906 \cdot 10^{7} \\
I x z=6.409 \cdot 10^{6}\end{array}$ & $\begin{array}{l}I x x=4.690 \cdot 10^{7} \\
I y y=7.100 \cdot 10^{7} \\
I z z=5.835 \cdot 10^{7} \\
I x y=1.392 \cdot 10^{7} \\
I y z=1.380 \cdot 10^{7} \\
I x z=6.409 \cdot 10^{6}\end{array}$ \\
\hline $\begin{array}{l}q_{1}=0 ; \quad q_{2}=120 ; \\
q_{3}=0 ; \quad q_{4}=120\end{array}$ & $\begin{array}{c}I x x=6.544 \cdot 10^{6} \\
I y y=1.407 \cdot 10^{7} \\
I z z=1.822 \cdot 10^{7} \\
I x y=0 \\
I y z=0 \\
I x z=0\end{array}$ & $\begin{array}{l}I x x=1.532 \cdot 10^{7} \\
I y y=5.123 \cdot 10^{7} \\
I z z=4.652 \cdot 10^{7} \\
I x y=1.384 \cdot 10^{7} \\
I y z=2.906 \cdot 10^{7} \\
I x z=6.448 \cdot 10^{6}\end{array}$ & $\begin{array}{l}I x x=2.894 \cdot 10^{7} \\
I y y=6.787 \cdot 10^{7} \\
I z z=4.954 \cdot 10^{7} \\
I x y=1.384 \cdot 10^{7} \\
I y z=2.906 \cdot 10^{7} \\
I x z=2.931 \cdot 10^{7}\end{array}$ \\
\hline $\begin{array}{l}q_{1}=90 ; q_{2}=120 ; \\
q_{3}=90 ; q_{4}=120\end{array}$ & $\begin{array}{c}I x x=6.544 \cdot 10^{6} \\
I y y=1.407 \cdot 10^{7} \\
I z z=1.822 \cdot 10^{7} \\
I x y=0 \\
I y z=0 \\
I x z=0\end{array}$ & $\begin{array}{l}I x x=1.531 \cdot 10^{7} \\
I y y=5.116 \cdot 10^{7} \\
I z z=4.652 \cdot 10^{7} \\
I x y=1.390 \cdot 10^{7} \\
I y z=2.905 \cdot 10^{7} \\
I x z=6.401 \cdot 10^{6}\end{array}$ & $\begin{array}{l}I x x=3.439 \cdot 10^{7} \\
I y y=6.821 \cdot 10^{7} \\
I z z=4.901 \cdot 10^{7} \\
I x y=1.318 \cdot 10^{7} \\
I y z=3.521 \cdot 10^{7} \\
I x z=4.456 \cdot 10^{6}\end{array}$ \\
\hline
\end{tabular}

Moments of inertia of the AMR of a changing configuration relative to the coupled coordinate system $M X_{M} Y_{M} Z_{M}\left(\mathrm{~g} \cdot \mathrm{cm} \mathrm{m}^{2}\right)$ depending on the values of the generalized coordinates of moving structural elements

Table 3

Moments of inertia of the AMR of a changing configuration relative

\begin{tabular}{|c|c|c|c|}
\hline $\begin{array}{c}\text { The value of } \\
\text { the generalized } \\
\text { coordinates of the } \\
\text { manipulator }\end{array}$ & $\begin{array}{c}\text { Loading weight } \\
m_{l}=4000 \mathrm{~g} \\
\text { Platform weight } \\
m_{P}=50625 \mathrm{~g}\end{array}$ & $\begin{array}{c}\text { Loading weight } \\
m_{l}=6000 \mathrm{~g} \\
\text { Platform weight } \\
m_{P}=50625 \mathrm{~g}\end{array}$ & $\begin{array}{c}\text { Loading weight } \\
m_{l}=84000 \mathrm{~g} \\
\text { Platform weight } \\
m_{P}=50625 \mathrm{~g}\end{array}$ \\
\hline $\begin{array}{c}q_{1}=0 ; q_{2}=10 ; \\
q_{3}=0 ; q_{4}=0\end{array}$ & $\begin{array}{l}I x x=3.518 \cdot 10^{7} \\
I y y=8.282 \cdot 10^{7} \\
I z z=5.835 \cdot 10^{7} \\
I x y=1.384 \cdot 10^{7} \\
I y z=2.906 \cdot 10^{7} \\
I x z=2.168 \cdot 10^{7}\end{array}$ & $\begin{array}{c}I x x=4.51 \cdot 10^{7} \\
I y y=9.861 \cdot 10^{7} \\
I z z=6.421 \cdot 10^{7} \\
I x y=1.384 \cdot 10^{7} \\
I y z=2.906 \cdot 10^{7} \\
I x z=2.932 \cdot 10^{7}\end{array}$ & $\begin{array}{l}I x x=5.503 \cdot 10^{7} \\
I y y=1.144 \cdot 10^{7} \\
I z z=7.008 \cdot 10^{7} \\
I x y=1.384 \cdot 10^{7} \\
I y z=2.906 \cdot 10^{7} \\
I x z=3.695 \cdot 10^{7}\end{array}$ \\
\hline $\begin{array}{c}q_{1}=90 ; q_{2}=10 ; \\
q_{3}=0 ; q_{4}=0\end{array}$ & $\begin{array}{l}I x x=4.690 \cdot 10^{7} \\
I y y=7.100 \cdot 10^{7} \\
I z z=5.835 \cdot 10^{7} \\
I x y=1.392 \cdot 10^{7} \\
I y z=1.380 \cdot 10^{7} \\
I x z=6.409 \cdot 10^{6}\end{array}$ & $\begin{array}{l}I x x=6.269 \cdot 10^{7} \\
I y y=8.093 \cdot 10^{7} \\
I z z=6.421 \cdot 10^{7} \\
I x y=1.392 \cdot 10^{7} \\
I y z=6.167 \cdot 10^{6} \\
I x z=6.409 \cdot 10^{6}\end{array}$ & $\begin{array}{c}I x x=7.849 \cdot 10^{7} \\
I y y=9.085 \cdot 10^{7} \\
I z z=7.008 \cdot 10^{7} \\
I x y=1.392 \cdot 10^{7} \\
I y z=-1.465 \cdot 10^{6} \\
I x z=6.409 \cdot 10^{6}\end{array}$ \\
\hline $\begin{array}{l}q_{1}=0 ; q_{2}=120 ; \\
q_{3}=0 ; q_{4}=120\end{array}$ & $\begin{array}{l}I x x=2.894 \cdot 10^{7} \\
I y y=6.787 \cdot 10^{7} \\
I z z=4.954 \cdot 10^{7} \\
I y z=1.384 \cdot 10^{7} \\
I y z=2.906 \cdot 10^{7} \\
I_{x z}=2.931 \cdot 10^{7}\end{array}$ & $\begin{array}{l}I x x=3.575 \cdot 10^{7} \\
I y y=7.619 \cdot 10^{7} \\
I z z=5.105 \cdot 10^{7} \\
I x y=1.384 \cdot 10^{7} \\
I y z=2.906 \cdot 10^{7} \\
I x z=-3.180 \cdot 10^{6}\end{array}$ & $\begin{array}{l}I x x=4.256 \cdot 10^{7} \\
I y y=8.451 \cdot 10^{7} \\
I z z=5.257 \cdot 10^{7} \\
I x y=1.384 \cdot 10^{7} \\
I y z=2.906 \cdot 10^{7} \\
I x z=-6.389 \cdot 10^{7}\end{array}$ \\
\hline $\begin{array}{l}q_{1}=90 ; q_{2}=120 ; \\
q_{3}=90 ; q_{4}=120\end{array}$ & $\begin{array}{l}I x x=3.439 \cdot 10^{7} \\
I y y=6.821 \cdot 10^{7} \\
I z z=4.901 \cdot 10^{7} \\
I x y=1.318 \cdot 10^{7} \\
I y z=3.521 \cdot 10^{7} \\
I x z=4.456 \cdot 10^{6}\end{array}$ & $\begin{array}{l}I x x=4.392 \cdot 10^{7} \\
I y y=7.672 \cdot 10^{7} \\
I z z=5.025 \cdot 10^{7} \\
I x y=1.283 \cdot 10^{7} \\
I y z=3.829 \cdot 10^{7} \\
I x z=3.483 \cdot 10^{6}\end{array}$ & $\begin{array}{l}I x x=5.346 \cdot 10^{7} \\
I y y=8.525 \cdot 10^{7} \\
I z z=5.149 \cdot 10^{7} \\
I x y=1.247 \cdot 10^{7} \\
I y z=4.137 \cdot 10^{7} \\
I x z=2.510 \cdot 10^{6}\end{array}$ \\
\hline
\end{tabular}
to the coupled coordinate system $M X_{M} Y_{M} Z_{M}\left(\mathrm{~g} \cdot \mathrm{cm}^{2}\right)$ depending on the ratio of the mass of the AMR platform and the mass of the moving structural elements 
The moments of inertia of the AMR of a changing configuration with respect to the axes of the base coordinate system $M X_{M} Y_{M} Z_{M}$ depend non-linearly and disproportionately on the ratio of the mass of the AMR platform and the mass of the moving elements of the structure. Thus, at $q_{1}=0, q_{2}=120, q_{3}=0, q_{4}=120$, an increase in the load mass does not change the values of the centrifugal moments of inertia: Ixy=const and Iyz=const (Table 3). At $q_{1}=90, q_{2}=120, q_{3}=90, q_{4}=120$, an increase in the load mass corresponds to a decrease in the values of the centrifugal moments $I_{x y}$ and $I_{x z}$ (Table 3 ).

It should be noted that the above results were obtained under certain assumptions, namely: the links of the manipulator and the platform are absolutely rigid and homogeneous bodies with a stable density; let's neglect the mass of kinematic pairs. The mass of moving structural elements, i. e., a manipulator with a load, is 10-20\% of the mass of the robot platform. The next step in the analysis of the AMR inertia tensor of a changing configuration can be a study taking into account the elastic properties of the manipulator links, the uneven distribution of the masses of the platform, and the characteristics of the kinematic pairs.

\section{Conclusions}

The inertia tensor analysis contains:

- calculation of axial and centrifugal moments of inertia of each link in coupled coordinate systems $O_{i} X_{i} Y_{i} Z_{i}$ $(i=1,2,3,4)$;

- determination of the elements of the inertia tensor of the transferred bodies in the base coordinate system $M X_{M} Y_{M} Z_{M}$

- calculation in the base coordinate system $M X_{M} Y_{M} Z_{M}$ of values and estimation of increments of the elements of the AMR inertia tensor with a manipulator for different configurations of their relative positions;

- establishing the dependence of the values of the elements of the AMR inertia tensor of the changing configuration on the values of the generalized coordinates of the moving elements of the structure;

- establishing the dependence of the values of the elements of the AMR inertia tensor of a changing configuration on the ratio of the mass of the AMR platform and the mass of the moving structural elements.

Based on the data obtained, the analysis of the offdiagonality and non-stationarity of the inertia tensor of the AMR with a manipulator is made at different configurations of their mutual position. The values of the elements of the AMR tensor of the changing configuration depend non-linearly on the values of the generalized coordinates of the moving elements of the structure. The moments of inertia of the AMR of a changing configuration with respect to the axes of the base coordinate system $M X_{M} Y_{M} Z_{M}$ depend non-linearly and disproportionately on the ratio of the mass of the AMR platform and the mass of the moving elements of the structure. A feature of objects of this class is the commensurability of the centrifugal and axial moments of inertia relative to the axes of the coordinate system coupled with the AMR platform, even if the mass of moving structural elements is less than $10 \%$ of the platform mass.

The analysis of the inertia tensor of the AMR with a manipulator at different configurations of their mutual position showed that the values of the centrifugal moments of inertia of the system during the relative motion of the manipulator are commensurate with the value of the axial moments of inertia of the system. Therefore, in the general case, the inertia tensor of such a system should be taken as off-diagonal and non-stationary.

In the future, this will make it possible to refine the equation of dynamics, determine the relationship of control channels, simulate the movement of an AMR with a manipulator, and optimize energy consumption. The presence of off-diagonal elements of the inertia tensor leads to the rotation of the AMR of a changing configuration around the axes of the base coordinate system $M X_{M} Y_{M} Z_{M}$ from the control moment applied along one axis of the base coordinate system.

Since AMR with the manipulator is an example of the «changing AMR» object class, the results obtained can be applied to all objects of this class.

\section{References}

1. Lopota, A., Spassky, B. (2020). Mobile ground-based robot systems for professional use. Robotics and Technical Cybernetics, 8 (1), 5-17. doi: http://doi.org/10.31776/rtcj.8101

2. Tsarichenko, S., Antokhin, E., Chernova, P., Dementey, V. (2020) The state and problems of standardization and unification of military ground robot systems. Robotics and Technical Cybernetics, 8 (1), 18-23. doi: https://doi.org/10.31776/rtcj.8102

3. Ermolov, I. L., Khripunov, S. P., Blagodariaschev, I. V., Khripunov, S. S. (2017). Tipovaia strukturno-funktsionalnaia skhema robototekhnicheskikh kompleksov voennogo naznacheniia. Informatsionno-izmeritelnie i upravliaiuschie sistemi, 15 (6), 4-9. Available at: https://elibrary.ru/item.asp?id=29712433

4. Stravopodis, N. A., Katrantzis, L., Moulianitis, V. C., Valsamos, C., Aspragathos, N. A.; Zeghloul, S., Laribi, M. A., Sebastian, J., Arevalo, S. (Eds.) (2020). Evaluation of Serial Metamorphic Manipulator Structures Considering Inertia Characteristics. Advances in Service and Industrial Robotics. Cham: Springer, 574-587. doi: http://doi.org/10.1007/978-3-030-48989-2_61

5. Wang, Y., Dehio, N., Kheddar, A. (2022). On Inverse Inertia Matrix and Contact-Force Model for Robotic Manipulators at Normal Impacts. IEEE Robotics and Automation Letters, 1-1. doi: http://doi.org/10.1109/lra.2022.3145967

6. Ashchepkova, N. S. (2013). Modeli i metod rozrakhunku vytrat enerhii na upravlinnia kutovym rukhom kosmichnoho aparatu zminiuvanoi konfihuratsii. Kharkiv: NAU KhAI, 187.

7. Fan, Y., Jing, W. (2021). Inertia-free appointed-time prescribed performance tracking control for space manipulator. Aerospace Science and Technology, 117, 106896. doi: http://doi.org/10.1016/ j.ast.2021.106896

8. Rubtsov, V. I., Mashkov, K. J., Konovalov, K. V. (2021). MultiLevel Control System for an Intelligent Robot that is Part of a Group. Mekhatronika, Avtomatizatsiya, Upravlenie, 22 (11), 610-615. doi: http://doi.org/10.17587/mau.22.610-615

9. Kurochkin, S. Y., Tachkov, A. A. (2021). Methods of Formation Control for a Group of Mobile Robots (a Review). Mekhatronika, Avtomatizatsiya, Upravlenie, 22 (6), 304-312. doi: http://doi.org/ 10.17587/mau.22.304-312

10. Kenzin, M., Bychkov, I., Maksimkin, N. (2020). Coordinated Recharging of Heterogeneous Mobile Robot Teams during Continuous Large Scale Missions. 2020 7th International Conference on Control, Decision and Information Technologies (CoDIT), 745-750. doi: http://doi.org/10.1109/codit49905.2020.9263974

11. Berezin, L. M., Koshel, S. O. (2019). Teoretychna mekhanika. Kyiv: Tsentr navchalnoi literatury, 218.

12. Iurevich, E. I. (2017). Osnovy robototekhniki. Saint Petersburg: BKHV-Peterburg, 304.

Natalja Ashhepkova, PhD, Associate Professor, Department of Mechanotronics, Oles Honchar Dnipro National University, Dnipro, Ukraine, e-mail: ashchepkovanatalya@gmail.com, ORCID: http:// orcid.org/0000-0002-1870-1062 